\title{
AN $S_{3}$-SYMMETRIC LITTLEWOOD-RICHARDSON RULE
}

\author{
Hugh Thomas And Alexander Yong
}

\section{Introduction}

Fix Young shapes $\lambda, \mu, \nu \subseteq \Lambda:=\ell \times k$. Viewing the Littlewood-Richardson coefficient $C_{\lambda, \mu, v}$ as the intersection number of generic translates of the Schubert varieties $X_{\lambda}, X_{\mu}, X_{v}$ in the Grassmannian of $\ell$-dimensional planes in $\mathbb{C}^{\ell+k}$, one has the obvious $S_{3}$-symmetries:

$$
\mathrm{C}_{\lambda, \mu, v}=\mathrm{C}_{\mu, \nu, \lambda}=\mathrm{C}_{\nu, \lambda, \mu}=\mathrm{C}_{\mu, \lambda, v}=\mathrm{C}_{\nu, \mu, \lambda}=\mathrm{C}_{\lambda, \nu, \mu} .
$$

There is interest in the combinatorics of these symmetries. The LittlewoodRichardson coefficients appear in a variety of contexts in addition to the Schubert calculus context described above, such as the representation theory of the symmetric group/complex general linear groups; see, e.g., [Fu97]. Combinatorially, $C_{\lambda, \mu, \nu}$ arises as follows: Let $s_{\lambda}:=s_{\lambda}\left(x_{1}, x_{2}, \ldots x_{\ell}\right)$ be the Schur polynomial indexed by $\lambda$; see, e.g., [St99, Chapter 7]. These form a $\mathbb{Z}$-linear basis of the ring $\Lambda\left(x_{1}, \ldots, x_{\ell}\right)$ of symmetric polynomials in the variables $x_{1}, \ldots, x_{\ell}$ (when $\lambda$ ranges over all Young shapes with at most $\ell$ rows), and the Littlewood-Richardson coefficients are the structure constants with respect to this basis. Moreover, $C_{\lambda, \mu, \nu}$ is the coefficient of $s_{\ell \times k}$ in the expansion of $s_{\lambda} s_{\mu} s_{\nu}$ in this basis, and thus $C_{\lambda, \mu, v}$ is clearly symmetric in the input data $\lambda, \mu, v$. However, previously studied Littlewood-Richardson rules for $C_{\lambda, \mu, v}$ manifest at most three of the six symmetries, see, e.g., the original rule proposed by [LiRi34] as well as work of [BeZe91, KnTaWo01, VaPa05, HeKa06], and the references therein.

We construct a carton rule for $C_{\lambda, \mu, v}$ that transparently and uniformly explains all of the symmetries (1).

Figure 1 depicts a carton, i.e., a three-dimensional box with a grid drawn rectilinearly on the six faces of its surface. Along the " $\emptyset, T_{\lambda}, T_{\mu}$ " face, the grid has $(|\mu|+1) \times(|\lambda|+1)$ vertices (including ones on the bounding edges of the face); the remainder of the grid is similarly determined. Define a carton filling to be an assignment of a Young diagram to each vertex of the grid so the shapes increase one box at a time while moving away from $\emptyset$, and so that, for any subgrid $\begin{array}{r}\alpha-\beta \\ \gamma-\delta\end{array}$

\section{Fomin growth assumptions hold:}

(F1) if $\alpha$ is the unique shape containing $\gamma$ and contained in $\beta$, then $\delta=\alpha$;

(F2) otherwise there is a unique such shape other than $\alpha$, and this shape is $\delta$.

Notice that these conditions are symmetric in $\alpha$ and $\delta$.

Received by the editors May 20, 2007. 
Initially, assign the shapes $\emptyset$ and $\Lambda$ to opposite corners, as in Figure 1. A standard Young tableau $T \in S Y T(\sigma / \pi)$ of shape $\sigma / \pi$ is equivalent to a shape chain in Young's lattice, e.g.,

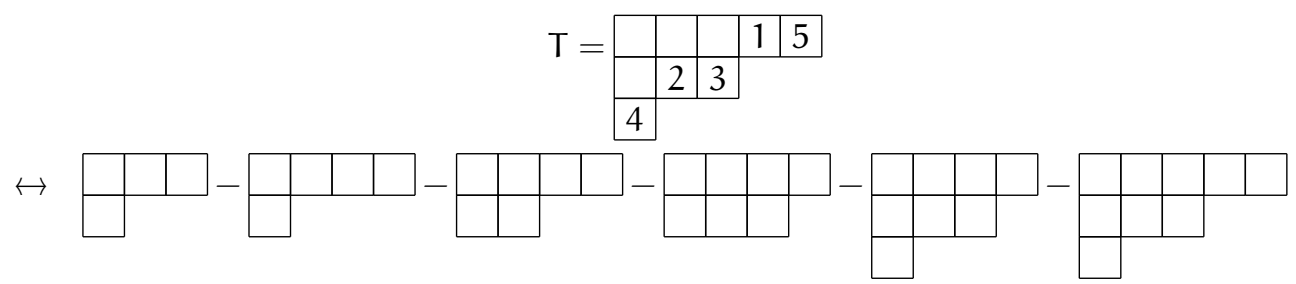

by starting with the unfilled inner shape, and adding one box at a time to indicate the box containing 1, then 2, etc. Fix a choice of standard tableaux $T_{\lambda}, T_{\mu}$ and $T_{\nu}$ of respective shapes $\lambda, \mu$ and $\nu$. Initialize the indicated edges with the shape chains for these tableaux.

Let CARTONS $_{\lambda, \mu, v}$ be all carton fillings with the above initial data.

Main Theorem. The Littlewood-Richardson coefficient $\mathrm{C}_{\lambda, \mu, v}$ equals \#CARTONS ${ }_{\lambda, \mu, v}$.

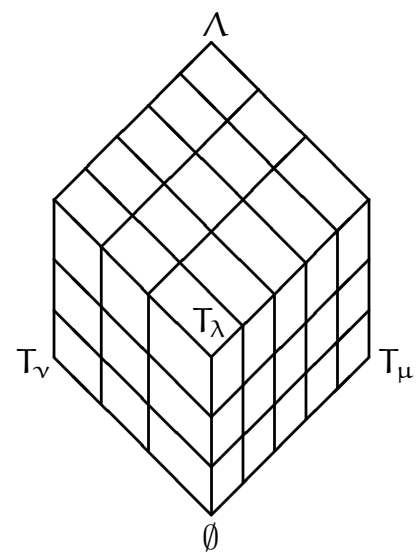

Figure 1. The carton rule counts $C_{\lambda, \mu, \nu}$ by assigning Young diagrams to the vertices of the six faces

This rule manifests bijections between CARTONS $_{\lambda, \mu, v}$ and CARTONS $_{\delta, \epsilon, \zeta}$ for permutations $(\delta, \epsilon, \zeta)$ of $(\lambda, \mu, v)$. We remark that it can be stated as counting lattice points of a 0,1-polytope, and readily extends to the setting of [ThYo06, ThYo07].

The Theorem is proved in Section 2, starting from the jeu de taquin formulation of the Littlewood-Richardson rule, and using Fomin's growth diagram ideas. In Figure 2 we give an example of the Main Theorem. An extended example is given in Section 3.

\section{Tableau facts and proof of the Main Theorem}

2.1. Tableau sliding. There is a partial order $\prec$ on the rectangle $\Lambda$ where $x \prec y$ if $x$ is weakly northwest of $y$. Given $T \in \operatorname{SYT}(\nu / \lambda)$ consider $x \in \lambda$, maximal in $\prec$ subject to being less than some box of $v / \lambda$. Associate another standard tableau 


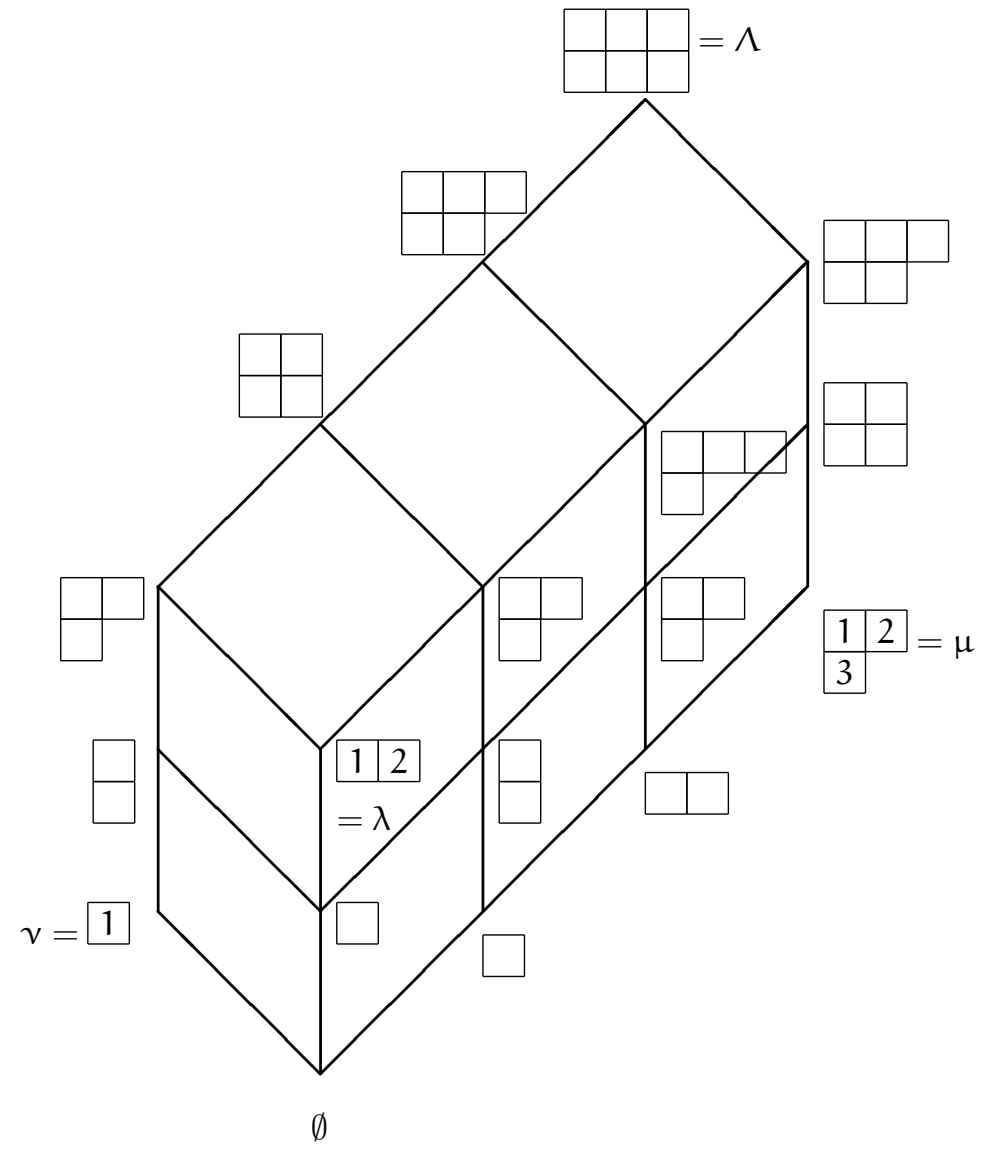

Figure 2. The "front" three faces of a carton filling for $C_{(2),(2,1),(1)}=1$. The choices of tableaux $T_{\lambda}, T_{\mu}$ and $T_{\nu}$ are as shown.

$j \operatorname{dt}_{x}(T)$, called the jeu de taquin slide of T into $x$ : Let $y$ be the box of $v / \lambda$ with the smallest label, among those covering $x$. Move the label of $y$ to $x$, leaving $y$ vacant. Look for boxes of $v / \lambda$ covering $y$ and repeat, moving into $y$ the smallest label among those boxes covering it. Then $j \operatorname{jdt}_{x}(T)$ results when no further slides are possible. The rectification of $T$ is the iteration of jeu de taquin slides until terminating at a straight shape standard tableau rectification $(\mathrm{T})$.

We can impose a specific "inner" $U \in \operatorname{SYT}(\lambda)$ that encodes the order in which the jeu de taquin slides are done. For example, if $U=$\begin{tabular}{|l|l|l|}
\hline 1 & 2 & 3 \\
\hline 4 & & then
\end{tabular}

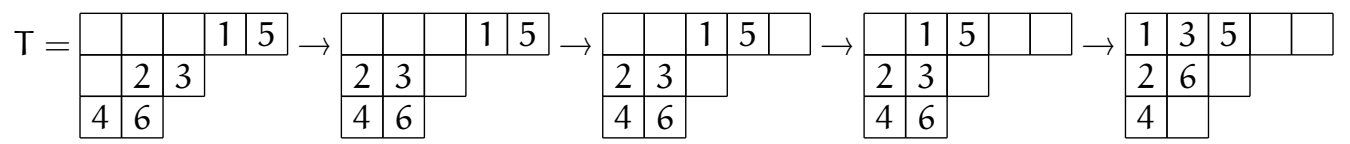


= rectification(T). Placing these chains one atop another (starting with T's and ending with rectification( $\mathrm{T}$ )'s) gives a Fomin growth diagram, which in this case is given in Table 1. Growth diagrams satisfy (F1) and (F2); see Fomin's [St99, Appendix 2] for more.

\begin{tabular}{|l|l|l|l|l|l|l|}
\hline$(3,1)$ & $(4,1)$ & $(4,2)$ & $(4,3)$ & $(4,3,1)$ & $(5,3,1)$ & $(5,3,2)$ \\
\hline$(3)$ & $(4)$ & $(4,1)$ & $(4,2)$ & $(4,2,1)$ & $(5,2,1)$ & $(5,2,2)$ \\
\hline$(2)$ & $(3)$ & $(3,1)$ & $(3,2)$ & $(3,2,1)$ & $(4,2,1)$ & $(4,2,2)$ \\
\hline$(1)$ & $(2)$ & $(2,1)$ & $(2,2)$ & $(2,2,1)$ & $(3,2,1)$ & $(3,2,2)$ \\
\hline$\emptyset$ & $(1)$ & $(1,1)$ & $(2,1)$ & $(2,1,1)$ & $(3,1,1)$ & $(3,2,1)$ \\
\hline
\end{tabular}

TABLE 1. A growth diagram

Given a top row $\mathrm{U}$ and left column $\mathrm{T}$, define $\operatorname{infusion}_{1}(\mathrm{U}, \mathrm{T})$ to be the bottom row of the growth diagram, and infusion $2(U, T)$ to be the right column. We write infusion $(U, T)$ for the ordered pair (infusion $(U, T)$, infusion $2(U, T)$ ). Growth diagrams are transpose symmetric, because (F1) and (F2) are. So one has the infusion involution:

$$
\text { infusion(infusion }(\mathrm{U}, \mathrm{T}))=(\mathrm{U}, \mathrm{T}) \text {. }
$$

If $\mathrm{U}$ is a straight shape, infusion $(\mathrm{U}, \mathrm{T})=\operatorname{rectification}(\mathrm{T})$, while infusion $_{2}(U, T)$ encodes the order in which squares were vacated in the jeu de taquin process.

Also, given $T \in \operatorname{SYT}(\nu / \lambda)$, consider $x \in \Lambda \backslash \nu$ minimal subject to being larger than some element of $v / \lambda$. The reverse jeu de taquin slide $\operatorname{revjdt}_{x}(T)$ of $T$ into $x$ is defined similarly to a jeu de taquin slide, except we move into $x$ the largest of the labels among boxes in $v / \lambda$ covered by $x$. We define reverse rectification revrectification $(T)$ similarly. The first fundamental theorem of jeu de taquin asserts (rev)rectification is well-defined.

Fix $T_{\mu} \in \operatorname{SYT}(\mu)$. The number of $T \in \operatorname{SYT}(\nu / \lambda)$ such that rectification $(T)=$ $T_{\mu}$ equals $C_{\lambda, \mu, v} v=C_{\lambda, \mu}^{v}$ where $v^{\vee}$ is the straight shape obtained as the 180-degree rotation of $\Lambda \backslash v$. This is Schützenberger's jeu de taquin formulation of the LittlewoodRichardson rule, see [St99, Appendix 2].

By a slide we mean either kind of jeu de taquin slide. Consider two equivalence relations on a pair of tableaux $\mathrm{T}$ and $\mathrm{U}$. Tableaux are jeu de taquin equivalent if one can be obtained from the other by a sequence of slides. They are dual equivalent if any such sequence results in tableaux of the same shape [Ha92]. Facts: Tableaux of the same straight shape are dual equivalent. A common application of slides to dual equivalent tableaux produces dual equivalent tableaux. A pair of tableaux that are both jeu de taquin and dual equivalent must be equal.

Recall Schützenberger's evacuation map. For $\mathrm{T} \in \mathrm{SYT}(\lambda)$, let $\uparrow$ be obtained by erasing the entry 1 (in the northwest corner $c$ ) of $T$ and subtracting 1 from the remaining entries. Let $\Delta(T)=j d t_{c}(\hat{T})$. The evacuation evac $(T) \in \operatorname{SYT}(\lambda)$ is defined by the shape chain

$$
\emptyset=\operatorname{shape}\left(\Delta^{|\lambda|}(T)\right)-\operatorname{shape}\left(\Delta^{|\lambda|-1}(T)\right)-\ldots-\operatorname{shape}\left(\Delta^{1}(T)\right)-T .
$$

This map is an involution: $\operatorname{evac}(\operatorname{evac}(\mathrm{T}))=\mathrm{T}$. 
2.2. Proof of the rule. Given $T \in \operatorname{SYT}(\alpha)$ for a straight shape $\alpha$, define $\widetilde{T} \in$ $\operatorname{SYT}(\operatorname{rotate}(\alpha))$ where rotate $(\alpha)=\Lambda \backslash \alpha^{\vee}$ by computing evac $(\mathrm{T}) \in \operatorname{SYT}(\alpha)$, replacing entry $i$ with $|\alpha|-i+1$ throughout and rotating the resulting tableau 180-degrees and placing it at the bottom right corner of $\Lambda$.

We need the following well-known fact. The proof we give extends straightforwardly to the setup of [ThYo06, ThYo07], allowing a "cominuscule" version of the Main Theorem.

Lemma 2.1. Suppose $\alpha, \beta$ and $\gamma$ are shapes where

$$
\begin{aligned}
& \quad \mathrm{T}_{\beta} \in \operatorname{SYT}(\beta), \mathrm{T}_{\gamma^{\vee} / \alpha} \in \operatorname{SYT}\left(\gamma^{\vee} / \alpha\right) \text { and } \operatorname{rectification}\left(\mathrm{T}_{\gamma^{\vee} / \alpha}\right)=\mathrm{T}_{\beta} . \\
& \text { Then revrectification }\left(\mathrm{T}_{\gamma^{\vee} / \alpha}\right)=\text { revrectification }\left(\mathrm{T}_{\beta}\right)=\widetilde{\mathrm{T}}_{\beta} .
\end{aligned}
$$

Proof. It suffices to show that revrectification $\left(\mathrm{T}_{\beta}\right)=\widetilde{\mathrm{T}}_{\beta}$. We induct on $|\beta|=n$.

Let $\mathrm{U}$ be the tableau obtained by removing the box labeled 1 from $T_{\beta}$. So $\mathrm{U}$ is of skew shape $\beta /(1)$. Let $V=\operatorname{rectification}(U)$, a tableau of shape $\kappa \subset$ $\beta$. By induction, revrectification $(V)=\widetilde{V}$. Now revrectification $\left(T_{\beta}\right)$ and revrectification(V) agree except that the former has a label 1 in a box that does not appear in the latter. In [ThYo06, Proposition 4.6], we showed that the reverse rectification of a tableau of shape $\beta$ is necessarily of shape rotate $(\beta)$. Thus, the location of 1 in revrectification $\left(T_{\beta}\right)$ must be the box $\kappa^{\vee} / \beta^{\vee}$. This is the 180 degree rotation of the box $\beta / \kappa$ in $\Lambda$, the latter being the position of $n$ in evac $\left(T_{\beta}\right)$. Thus, 1 is located in the desired position in revrectification $\left(T_{\beta}\right)$. The rest of the statement follows from the fact that the rest of revrectification $\left(T_{\beta}\right)$ agrees with $\widetilde{V}$, and the entries of evac $\left(T_{\beta}\right)$ other than $n$ agree with evac $(V)$.

Corollary 2.2. Fix a carton filling. The uninitialized corners of the " $\emptyset, \mathrm{T}_{\lambda}, \mathrm{T}_{\mu}$ " and " $\emptyset, \mathrm{T}_{\nu}, \mathrm{T}_{\lambda}$ " faces are $\mathrm{v}^{\vee}$ and $\mu \vee$ respectively. The remaining "sixth" corner (the unique one not visible in Figure 1) is $\lambda^{\vee}$. Thus, we can speak of the edges $\lambda^{\vee}-\Lambda$, $\mu^{\vee}-\Lambda$ and $\nu^{\vee}-\Lambda$. These represent the shape chains of $\widetilde{\mathrm{T}}_{\lambda}, \widetilde{\mathrm{T}}_{\mu}$ and $\widetilde{\mathrm{T}}_{\nu}$ respectively.

Proof. Think of the union of the faces " $\emptyset, T_{\mu}, T_{\lambda}$ " with the adjacent face involving $\Lambda$ and the "sixth corner" as a single growth diagram. This diagram computes revrectification $\left(T_{\lambda}\right)$ and records the result along the edge involving $\Lambda$ and diagonally opposite to the $T_{\lambda}$ edge. But the lemma asserts this result is $\widetilde{T}_{\lambda}$ and hence the "sixth corner vertex" is assigned $\lambda^{\vee}$. The other conclusions are proved similarly.

Corollary 2.2 affords us the convenience of referring to a face by its corner vertices. Note any carton filling gives a growth diagram on the face $\emptyset-\mu-v^{\vee}-\lambda$ for which the edge $\lambda-\nu^{\vee}$ is a standard tableau of shape $\nu^{\vee} / \lambda$ rectifying to $T_{\mu}$. By the jeu de taquin Littlewood-Richardson rule, fillings of this face count $C_{\lambda, \mu, v}$. Hence it suffices to show that any such growth diagram for this face extends uniquely to a filling of the entire carton.

If such an extension exists, it is unique: $\lambda-v^{\vee}$ and $\Lambda-v^{\vee}$ determine, by (F1), (F2) and the Corollary, the face $\lambda-\mu^{\vee}-\Lambda-v^{\vee}$. Similarly, these two faces determine the remaining faces (in order):

$\emptyset-v-\mu^{\vee}-\lambda \Longrightarrow v-\lambda^{\vee}-\Lambda-\mu^{\vee} \Longrightarrow \mu-\lambda^{\vee}-\Lambda-v^{\vee} \Longrightarrow \emptyset-v-\lambda^{\vee}-\mu$. 
We now show that with a filling of $\emptyset-\mu-v^{\vee}-\lambda$ (together with the extra edges given in the Corollary) one can extend it to fill the carton, using the conclusions of Corollary 2.2.

$\emptyset-\mu-v^{\vee}-\lambda$ : This is given by labeling $\lambda-v^{\vee}$ with $T_{v^{\vee} / \lambda} \in \operatorname{SYT}\left(v^{\vee} / \lambda\right)$ that $\overline{\text { witnesses } C_{\lambda, \mu, v}}=C_{\lambda, \mu}^{v^{\vee}}$, i.e., it rectifies to $T_{\mu}$. By the infusion involution, the edge $\mu-v^{\vee}$ representing $T_{\nu \vee / \mu}:=\operatorname{infusion}\left(T_{\lambda}, T_{\nu \vee / \lambda}\right) \in \operatorname{SYT}\left(\nu^{\vee} / \mu\right)$ witnesses $C_{\mu, \lambda}^{v^{\vee}}$.

$\lambda-\mu^{\vee}-\Lambda-v^{\vee}$ : Build the growth diagram using $T_{v^{\vee} / \lambda}$ and $\widetilde{T}_{v}$ from the edges $\lambda-v^{\vee}$ and $v^{\vee}-\Lambda$ respectively. The only boundary condition we need to check is that infusion $2\left(T_{v \vee / \lambda}, \widetilde{T}_{v}\right)=\widetilde{T}_{\mu}$, which is true by the Lemma. The newly determined edge $\lambda-\mu^{\vee}$ is infusion ${ }_{1}\left(T_{v} \vee / \lambda, \widetilde{T}_{v}\right)$. This is a tableau $T_{\mu} \vee / \lambda \in \operatorname{SYT}(\mu \vee / \lambda)$ which rectifies to $T_{v}$, i.e., one that witnesses $C_{\lambda, v}^{\mu^{\vee}}$.

$\emptyset-v-\mu^{\vee}-\lambda$ : Using the determined edges $\lambda-\mu^{\vee}$ and $\emptyset-\lambda$ we obtain a growth diagram with edge $\emptyset-v$ representing infusion ${ }_{1}\left(T_{\lambda}, T_{\mu} \vee / \lambda\right)$, which equals $T_{v}$, as desired. By the infusion involution, $v-\mu^{\vee}$ represents a tableau $T_{\mu} \vee / v \in \operatorname{SYT}\left(\mu^{\vee} / v\right)$ that witnesses $C_{v, \lambda}^{\mu \vee}$.

$v-\lambda^{\vee}-\Lambda-\mu^{\vee}$ : This time, we grow the face using the edges $v-\mu^{\vee}$ and $\mu^{\vee}-\Lambda$. The edge $\lambda^{\vee}-\Lambda$ is thus infusion $2\left(T_{\mu} \vee / v, \widetilde{T}_{\mu}\right)$ which indeed equals $\widetilde{T}_{\lambda}$, by the Lemma. The newly determined edge $v-\lambda^{\vee}$ is $T_{\lambda \vee / v}:=$ infusion $1\left(T_{\mu} \vee / v, \widetilde{T}_{\mu}\right) \in \operatorname{SYT}\left(\lambda^{\vee} / v\right)$ that witnesses $\mathrm{C}_{v, \mu}^{\lambda^{\vee}}$.

$\mu-\lambda^{\vee}-\Lambda-v^{\vee}$ : Growing the face using $\mu-v^{\vee}$ and $v^{\vee}-\Lambda$ we find that the edge $\overline{\lambda^{\vee}-\Lambda \text { equals infusion }}\left(\mathrm{T}_{v} \vee / \mu, \widetilde{T}_{v}\right)$, which is the already determined $\widetilde{T}_{\lambda}$. The newly determined edge $\mu-\lambda^{\vee}$ is $T_{\lambda \vee / \mu}:=\operatorname{infusion}\left(T_{v} \vee / \mu, \widetilde{T}_{v}\right) \in \operatorname{SYT}\left(\lambda^{\vee} / \mu\right)$ that witnesses $C_{\mu, v}^{\lambda^{v}}$.

$\emptyset-v-\lambda^{\vee}-\mu$ : We grow this final face using $\emptyset-v$ and $v-\lambda^{\vee}$, but need to make two consistency checks. First the edge $\emptyset-\mu$ is infusion ${ }_{1}\left(T_{v}, T_{\lambda} \vee / v\right)$ which clearly is $T_{\mu}$.

It remains to check that

$$
\text { infusion } 2\left(T_{v}, T_{\lambda \vee / v}\right)=T_{\lambda \vee / \mu} \text {. }
$$

(Notice that infusion $2\left(T_{v}, T_{\lambda} \vee / v\right)$ is a filling of $\lambda^{\vee} / \mu$ that rectifies to $T_{v}$, just as $\mathrm{T}_{\lambda \vee / \mu}$ does. However it is not clear a priori that they are the same.)

To prove (2), we need a definition. For tableaux $A$ and $B$ of respective (skew) shapes $\alpha$ and $\gamma / \alpha$ let $A \star B$ be their concatenation as a (nonstandard) tableau. If $C$ is a tableau of shape $\Lambda / \gamma$ and $\alpha$ is a straight shape, $A \star B \star C$ is a layered tableau of shape $\Lambda$.

Example 2.3. Let $\alpha=(2,1)$ and $\gamma=(4,2,1)$. We have

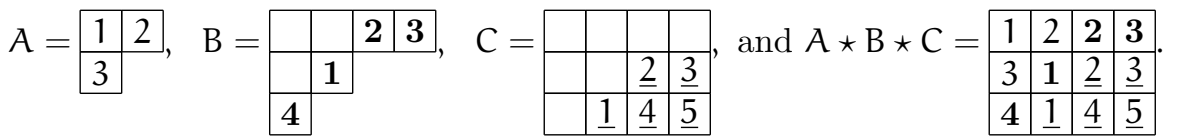

We have used boldface and underlining to distinguish the entries from $A, B$ and $C$. 
Let $\mathcal{I}_{1}$ and $\mathcal{I}_{2}$ be operators on layered tableaux defined by

$$
\mathcal{I}_{1}: A \star B \star C \mapsto \operatorname{infusion}_{1}(A, B) \star \operatorname{infusion}_{2}(A, B) \star C
$$

and

$$
\mathcal{I}_{2}: A \star B \star C \mapsto A \star \text { infusion }_{1}(B, C) \star \text { infusion }_{2}(B, C) .
$$

The infusion involution says $\mathcal{I}_{1}^{2}$ and $\mathcal{I}_{2}^{2}$ are the identity operator. In fact, the following crucial "braid identity" holds, showing $\mathcal{I}_{1}$ and $\mathcal{I}_{2}$ generate a representation of $\mathrm{S}_{3}$ :

Proposition 2.4. $\mathcal{I}_{1} \circ \mathcal{I}_{2} \circ \mathcal{I}_{1}=\mathcal{I}_{2} \circ \mathcal{I}_{1} \circ \mathcal{I}_{2}$.

In view of the Proposition, (2) follows by setting $\alpha=\lambda, \gamma=v^{\vee}, A=T_{\lambda}, B=T_{\lambda} \vee / v$ and $C=\widetilde{\mathrm{T}}_{\mathcal{V}}$, since the assertion merely says the "middle" tableau in

$$
\mathcal{I}_{1} \circ \mathcal{I}_{2} \circ \mathcal{I}_{1} \circ \mathcal{I}_{2}\left(T_{\lambda} \star T_{v \vee / \lambda} \star \widetilde{T}_{v}\right) \text { and } \mathcal{I}_{2} \circ \mathcal{I}_{1}\left(T_{\lambda} \star T_{v \vee / \lambda} \star \widetilde{T}_{v}\right)
$$

are the same, whereas we even have equality of the two layered tableaux.

Proof of Proposition 2.4: By the Lemma it follows that

$$
\begin{gathered}
\widetilde{C} \star \bar{B} \star \widetilde{A}:=\mathcal{I}_{1} \circ \mathcal{I}_{2} \circ \mathcal{I}_{1}(A \star B \star C), \text { and } \\
\widetilde{C} \star \widehat{B} \star \widetilde{A}:=\mathcal{I}_{2} \circ \mathcal{I}_{1} \circ \mathcal{I}_{2}(A \star B \star C),
\end{gathered}
$$

where the shapes of $\widetilde{C}, \bar{B}$ and $\widetilde{A}$ are respectively the 180 degree rotations of the shapes of $A, B$ and $C$, and we know the fillings of $\widetilde{C}$ and $\widetilde{A}$ in terms of evacuation. We thus also know the shapes of $\bar{B}$ and $\widehat{B}$ are the same. It remains to show $\bar{B}$ equals $\widehat{B}$.

We first study (4). Let $B^{\prime}=\operatorname{infusion}(A, B)$, and $A^{\prime}=\operatorname{infusion}(A, B)$. Now

$$
\mathcal{I}_{1} \circ \mathcal{I}_{2}\left(\mathrm{~B}^{\prime} \star A^{\prime} \star C\right)=\widetilde{C} \star \bar{B} \star \widetilde{A},
$$

and the skew tableau $\bar{B} \star \widetilde{A}$ is obtained by treating $B^{\prime} \star A^{\prime}$ as a single standard tableau by valuing an entry $i$ of $A^{\prime}$ as $\left|B^{\prime}\right|+i$, evacuating, rotating the result 180 degrees and finally sending the entry $i$ from $A^{\prime}$ (respectively $B^{\prime}$ ) to $\left|A^{\prime}\right|-i+1$ (respectively $\left.\left|B^{\prime}\right|-i+1\right)$.

Example 2.5. Continuing the previous example,

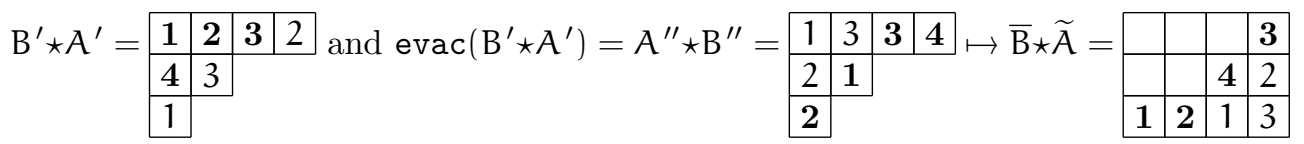

Let us focus on evac $\left(B^{\prime} \star A^{\prime}\right)=A^{\prime \prime} \star B^{\prime \prime}$ and momentarily ignore the subsequent rotation and complementation of entries. Fomin has shown in [St99, Appendix 2] that a fruitful way to think of evac $(X)$ is with triangular growth diagrams obtained by placing the shape chain of $X$, then $\Delta(X), \Delta^{2}(X), \ldots$ on top of one another (slanted left to right); see Figure 3.

We begin with the data $B^{\prime} \star A^{\prime}$ along the left side of the diagram. This is given as a concatenation of two shape chains, one from $\emptyset-\mu$ and then one from $\mu-\gamma$ where $\mu$ is the shape of $B^{\prime}$. Applying (F1) and (F2) from Section 1, we obtain $A^{\prime \prime} \star B^{\prime \prime}=\operatorname{evac}\left(B^{\prime} \star A^{\prime}\right)$, given along the righthand side as a pair of chains connected at $\alpha$. It follows from the construction of growth diagrams that the thick lines represent $\operatorname{evac}\left(B^{\prime}\right)$ and $A=\operatorname{evac}\left(A^{\prime \prime}\right)$ (the latter being a consequence of the Lemma). 


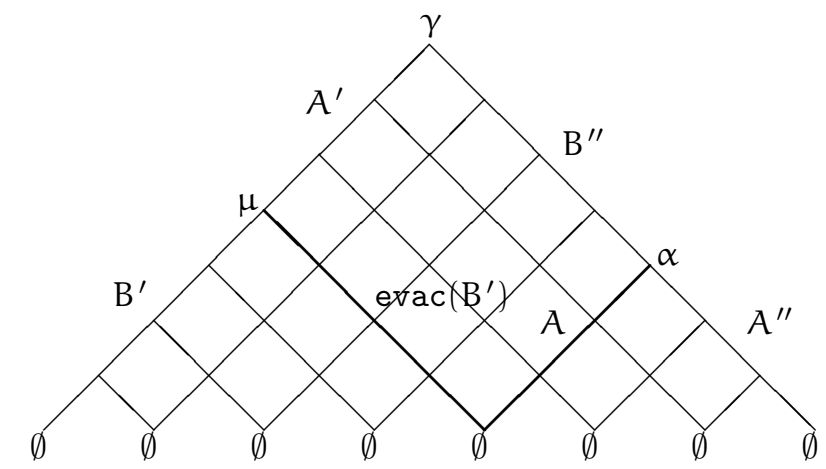

Figure 3. A triangular growth diagram to compute evac $\left(B^{\prime} \star A^{\prime}\right)$

These thick lines together with the vertex $\gamma$ define a rectangular growth diagram, so:

$$
\operatorname{infusion}\left(A, B^{\prime \prime}\right)=\left(\operatorname{evac}\left(B^{\prime}\right), A^{\prime}\right) \text {. }
$$

In particular, the shape of rectification $\left(B^{\prime \prime}\right)=\mu$. On the other hand, by definition

$$
\operatorname{infusion}(A, B)=\left(B^{\prime}, A^{\prime}\right) \text {, }
$$

and $B$ rectifies to a tableau of shape $\mu$ also.

By (7) and (8) combined with the aforementioned results of [Ha92] we see B and B" are dual equivalent, as they both are obtained by an application of the same sequence of slides (encoded by $A^{\prime}$ ) to a pair of tableaux of the same straight shape. Moreover, by (7) $B^{\prime \prime}$ is jeu de taquin equivalent to evac $\left(B^{\prime}\right)=$ evac(rectification $(B)$ ). Carrying out the analogous "reverse" analysis on $\mathcal{I}_{2} \circ \mathcal{I}_{1} \circ \mathcal{I}_{2}(A \star B \star C)$ we let $C^{\circ}=$ infusion $_{1}(B, C)$ and $B^{\circ}=$ infusion $_{2}(B, C)$ and study $\mathcal{I}_{2} \circ \mathcal{I}_{1}\left(A \star C^{\circ} \star B^{\circ}\right)=\widetilde{C} \star \widehat{B} \star \widetilde{A}$. Parallel to (6) we consider the "reverse evacuation" $\mathrm{B}^{\circ \circ} \star \mathrm{C}^{\circ \circ}$ of $\mathrm{C}^{\circ} \star \mathrm{B}^{\circ}$ using reverse jeu de taquin slides. Then we similarly conclude $\mathrm{B}^{\circ \circ}$ is dual equivalent to $\mathrm{B}$ (and thus $\mathrm{B}^{\prime \prime}$ ). Also $\mathrm{B}^{\circ \circ}$ is jeu de taquin equivalent to the reverse evacuation of revrectification(B). Thus by the lemma, $\mathrm{B}^{\prime \prime}$ and $\mathrm{B}^{\circ \circ}$ are jeu de taquin equivalent. Hence $\mathrm{B}^{\prime \prime}=\mathrm{B}^{\circ \circ}$ and so $\overline{\mathrm{B}}=\widehat{\mathrm{B}}$, as required.

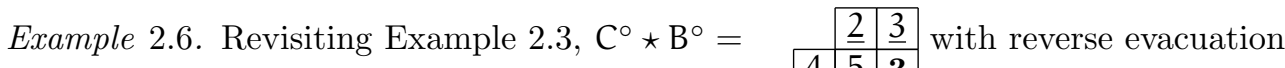
\begin{tabular}{|l|l|l|l|}
\cline { 2 - 4 } & $\underline{4}$ & $\underline{5}$ & 3 \\
\hline 1 & 1 & $\mathbf{2}$ & 4 \\
\hline
\end{tabular}

\begin{tabular}{|l|l|l|}
\hline $\mathbf{3}$ & $\mathbf{4}$ \\
\hline & 1 & 2
\end{tabular} , and $\mathrm{B}^{\circ \circ}$ (boldface in the latter tableau) is also $\mathrm{B}^{\prime \prime}$ from Example 2.5. \begin{tabular}{|l|l|l|l|l}
\hline 2 & 3 & 4 & 5 \\
\hline
\end{tabular}

Rotation and complementation gives $\widehat{\mathrm{B}}$, which is $\overline{\mathrm{B}}$ from Example 2.5, as desired. 


\section{An extended example of the main theorem}

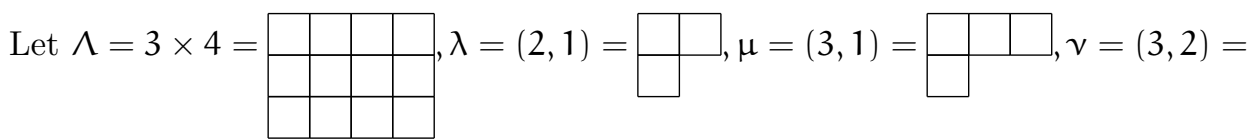
Therefore $\lambda^{\vee}=(4,3,2), \mu^{\vee}=(4,3,1)$ and $\nu^{\vee}=(4,2,1)$. Also

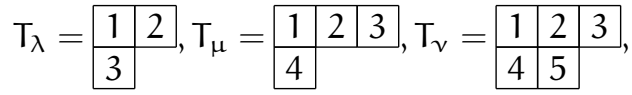

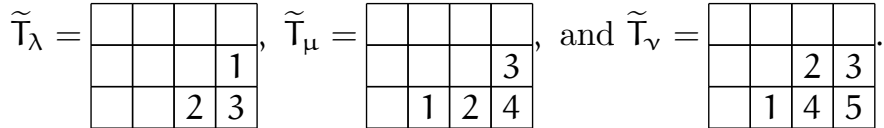

Here $C_{\lambda, \mu, v}=1$, and we now give the unique carton filling. We begin with $T_{\nu^{\vee} / \lambda}=$ \begin{tabular}{|l|l|l|l|l}
\hline & & 2 & 3 \\
\hline & 4 & &
\end{tabular} . Then we have the following sides of the carton, described as growth diagrams with the obvious identifications of boundaries (the partitions correspond to shapes placed on the vertices of the carton and the diagrams have been oriented to be consistent with Figure 1):

\begin{tabular}{|l|l|l|l|l|}
\hline$(2,1)=\lambda$ & $(2,1,1)$ & $(3,1,1)$ & $(4,1,1)$ & $(4,2,1)=v^{\vee}$ \\
\hline$(2)$ & $(2,1)$ & $(3,1)$ & $(4,1)$ & $(4,2)$ \\
\hline$(1)$ & $(1,1)$ & $(2,1)$ & $(3,1)$ & $(3,2)$ \\
\hline$\emptyset$ & $(1)$ & $(2)$ & $(3)$ & $(3,1)=\mu$ \\
\hline
\end{tabular}

TABLE $2 . \emptyset-\mu-v^{\vee}-\lambda$

\begin{tabular}{|l|l|l|l|l|}
\hline$(4,3,1)=\mu^{\vee}$ & $(4,3,2)$ & $(4,3,3)$ & $(4,4,3)$ & $(4,4,4)=\Lambda$ \\
\hline$(4,2,1)$ & $(4,2,2)$ & $(4,3,2)$ & $(4,4,2)$ & $(4,4,3)$ \\
\hline$(4,2)$ & $(4,2,1)$ & $(4,3,1)$ & $(4,4,1)$ & $(4,4,2)$ \\
\hline$(3,2)$ & $(3,2,1)$ & $(3,3,1)$ & $(4,3,1)$ & $(4,3,2)$ \\
\hline$(2,2)$ & $(2,2,1)$ & $(3,2,1)$ & $(4,2,1)$ & $(4,2,2)$ \\
\hline$(2,1)=\lambda$ & $(2,1,1)$ & $(3,1,1)$ & $(4,1,1)$ & $(4,2,1)=v^{\vee}$ \\
\hline
\end{tabular}

TABLE $3 . \lambda-\mu^{\vee}-\Lambda-\nu^{\vee}$

\begin{tabular}{|l|l|l|l|l|l|}
\hline$(4,3,1)=\mu^{\vee}$ & $(4,2,1)$ & $(4,2)$ & $(3,2)$ & $(2,2)$ & $(2,1)=\lambda$ \\
\hline$(4,2,1)$ & $(4,1,1)$ & $(4,1)$ & $(3,1)$ & $(2,1)$ & $(2)$ \\
\hline$(3,2,1)$ & $(3,1,1)$ & $(3,1)$ & $(2,1)$ & $(1,1)$ & $(1)$ \\
\hline$(3,2)=v$ & $(3,1)$ & $(3)$ & $(2)$ & $(1)$ & $\emptyset$ \\
\hline
\end{tabular}

TABLE $4 . \emptyset-v-\mu^{\vee}-\lambda$ 


\begin{tabular}{|l|l|l|l|l|}
\hline$(4,3,1)=\mu^{\vee}$ & $(4,3,2)$ & $(4,3,3)$ & $(4,4,3)$ & $(4,4,4)=\Lambda$ \\
\hline$(4,2,1)$ & $(4,2,2)$ & $(4,3,2)$ & $(4,4,2)$ & $(4,4,3)$ \\
\hline$(3,2,1)$ & $(3,2,2)$ & $(3,3,2)$ & $(4,3,2)$ & $(4,4,2)$ \\
\hline$(3,2)=\nu$ & $(3,2,1)$ & $(3,3,1)$ & $(4,3,1)$ & $(4,3,2)=\lambda^{\vee}$ \\
\hline
\end{tabular}

TABLE 5. $v-\lambda^{\vee}-\Lambda-\mu^{\vee}$

\begin{tabular}{|l|l|l|l|l|l|}
\hline$(4,4,4)=\Lambda$ & $(4,4,3)$ & $(4,4,2)$ & $(4,3,2)$ & $(4,2,2)$ & $(4,2,1)=v^{\vee}$ \\
\hline$(4,4,3)$ & $(4,4,2)$ & $(4,4,1)$ & $(4,3,1)$ & $(4,2,1)$ & $(4,2)$ \\
\hline$(4,3,3)$ & $(4,3,2)$ & $(4,3,1)$ & $(3,3,1)$ & $(3,2,1)$ & $(3,2)$ \\
\hline$(4,3,2)=\lambda^{\vee}$ & $(4,2,2)$ & $(4,2,1)$ & $(3,2,1)$ & $(3,1,1)$ & $(3,1)=\mu$ \\
\hline
\end{tabular}

TABLE $6 . \lambda^{\vee}-\Lambda-v^{\vee}-\mu$

\begin{tabular}{|l|l|l|l|l|}
\hline$(3,2)=v$ & $(3,2,1)$ & $(3,3,1)$ & $(4,3,1)$ & $(4,3,2)=\lambda^{\vee}$ \\
\hline$(3,1)$ & $(3,1,1)$ & $(3,2,1)$ & $(4,2,1)$ & $(4,2,2)$ \\
\hline$(3)$ & $(3,1)$ & $(3,2)$ & $(4,2)$ & $(4,2,1)$ \\
\hline$(2)$ & $(2,1)$ & $(2,2)$ & $(3,2)$ & $(3,2,1)$ \\
\hline$(1)$ & $(1,1)$ & $(2,1)$ & $(3,1)$ & $(3,1,1)$ \\
\hline$\emptyset$ & $(1)$ & $(2)$ & $(3)$ & $(3,1)=\mu$ \\
\hline
\end{tabular}

TABLE 7. $\emptyset-v-\lambda^{\vee}-\mu$

\section{Acknowledgments}

HT was partially supported by an NSERC Discovery Grant and AY by NSF grant 0601010. This work was partially completed while HT was a visitor at the Centre de Recherches Mathématiques and the University of Minnesota, and while AY was at the Fields Institute and the University of Michigan, Ann Arbor. We thank the Banff International Research Station for a common visit during the "Schubert calculus and Schubert geometry" workshop organized by Jim Carrell and Frank Sottile. We also thank Sergey Fomin, Allen Knutson, Ezra Miller, Vic Reiner, Luis Serrano, David Speyer and Dennis Stanton for helpful discussions.

\section{References}

[BeZe91] A. D. Berenstein and A. V. Zelevinsky, Triple multiplicities of $\mathrm{s} \ell(\mathrm{r}+1)$ and the spectrum of the exterior algebra of the adjoint representation, Journal of Algebraic Combinatorics, 1 (1992), 7-22.

[Fu97] W. Fulton, Young tableaux, London Mathematical Society Student Texts, 35. Cambridge University Press, Cambridge, 1997.

[Ha92] M. Haiman, Dual equivalence with applications, including a conjecture of Proctor, Discrete Math. 99 (1992), 79-113.

[HeKa06] A. Henriques and J. Kamnitzer, The octahedron recurrence and $\mathrm{gl}_{\mathrm{n}}$ crystals, Adv. Math., 206 (2006), no. 1, 211-249. 
[KnTaWo01] A. Knutson, T. Tao and C. Woodward, The honeycomb model of GL $(\mathfrak{n})$ tensor products II: Puzzles determine facets of the Littlewood-Richardson cone, J. Amer. Math. Soc., 17 (2004), 19-48.

[LiRi34] D. E. Littlewood and A. R. Richardson, Group characters and algebra, Phil. Trans. Royal. Soc. A (London) 233 (1934), 99-141.

[St99] R. P. Stanley, Enumerative Combinatorics, Volume 2 (with an appendix by S. Fomin), Cambridge University Press, 1999.

[VaPa05] E. Vallejo and I. Pak, Combinatorics and geometry of Littlewood-Richardson cones, Europ. J. Combinat., 26(2005), 995-1008.

[ThYo06] H. Thomas and A. Yong, A combinatorial rule for (co)minuscule Schubert calculus, preprint math.AG/0608273.

[ThYo07]_ Cominuscule tableau combinatorics, preprint math.CO/0701215.

Department of Mathematics and Statistics, University of New Brunswick, Fredericton, New Brunswick, E3B 5A3, CANada

E-mail address: hugh@math.unb.ca

Department of Mathematics, University of Minnesota, Minneapolis, MN 55455, USA, Department of Mathematics, University of Illinois at Urbana-Champaign, Urbana, IL 61801 (AFTER JulY 2008)

E-mail address: ayong@math.umn.edu 